\title{
MEAN CONVERGENCE OF JACOBI SERIES
}

\section{BENJAMIN MUCKENHOUPT ${ }^{1}$}

1. Introduction. The mean convergence of Jacobi series has been investigated by Pollard in [4] and Wing in [6]. Their results, however, are only valid if $\alpha \geqq-\frac{1}{2}$ and $\beta \geqq-\frac{1}{2}$ while Jacobi series are usually considered for all $\alpha$ and $\beta$ greater than -1 . The purpose of this paper is to fill this gap; the main result is the following.

Theorem 1. Assume that $\alpha>-1, \beta>-1,1<p<\infty$ and let $s_{n}{ }^{(\alpha, \beta)}(x)$ denote the nth partial sum of the Jacobi polynomial series for $f(x)$ with parameters $\alpha$ and $\beta$. Assume that $\left|a-\frac{1}{2}-\frac{1}{2} \alpha+1 / p\right|<\min \left(\frac{1}{4}, \frac{1}{2}+\frac{1}{2} \alpha\right)$ and that the same is true with $a$ and $\alpha$ replaced by $b$ and $\beta$. Then there exists a constant, $C$, independent of $f$ and $n$, such that

$$
\int_{-1}^{1}\left|s_{n}^{(\alpha, \beta)}(x)(1-x)^{a}(1+x)^{b}\right|^{p} d x \leqq C \int_{-1}^{1}\left|f(x)(1-x)^{a}(1+x)^{b}\right|^{p} d x .
$$

If $\alpha \geqq-\frac{1}{2}, \beta \geqq-\frac{1}{2}, a=\alpha / p$ and $b=\beta / p$, this is Pollard's result, and if $\alpha \geqq-\frac{1}{2}, \beta \geqq-\frac{1}{2}, a=\frac{1}{2} \alpha$ and $b=\frac{1}{2} \beta$, this is the Jacobi polynomial version of Wing's result. The mean convergence result follows from Theorem 1 and is as follows.

COROLlary. If $\alpha, \beta, a, b$ and $p$ satisfy the hypotheses of Theorem 1 and $f(x)$ is such that the right side of the conclusion of Theorem 1 is finite, then

$$
\lim _{n \rightarrow \infty} \int_{-1}^{1}\left|\left(s_{n}^{(\alpha, \beta)}(x)-f(x)\right)(1-x)^{a}(1+x)^{b}\right|^{p} d x=0 .
$$

These are also the best possible results in the following sense.

Theorem 2. If $\alpha>-1, \beta>-1,1<p<\infty$ and either a or $b$ does not satisfy the condition in Theorem 1, then the conclusions of Theorem 1 and the corollary are both false.

The general method of proof of Theorem 1 is to use the reduction derived by Pollard in [3] for the same purpose. This is combined with a weighted Hilbert transform inequality from [2] to produce the result. As usual, Theorem 2 is proved by estimating lower bounds of various integrals.

Received by the editors March 22, 1969.

1 Supported in part by N.S.F. Grant GP 7539. 
2. Results needed. The Jacobi polynomials, $P_{n}{ }^{(\alpha, \beta)}(x)$ will be defined as they are in [5]. (4.1.3), p. 59 of [5] states that

$$
P_{n}^{(\alpha, \beta)}(x)=(-1)^{n} P_{n}^{(\alpha, \beta)}(-x) .
$$

Theorem 7.32 .2 , p. 167 of [5] shows that there is a constant, $C$, independent of $x$ and $n$ such that

$$
\left|P_{n}^{(\alpha, \beta)}(x)\right| \leqq C n^{-1 / 2}\left(1-x+n^{-2}\right)^{-(1 / 2) \alpha-1 / 4}, \quad 0 \leqq x \leqq 1 .
$$

Theorem 8.21 .12 , p. 195 of [5] shows that there are positive constants, $C_{1}$ and $C_{2}$, independent of $n$ and $x$, such that for $n \geqq 1$

$$
\begin{aligned}
& \left|P_{n}^{(\alpha, \beta)}(x)\right| \geqq C_{1}(1-x)^{-(1 / 2) \alpha}\left|J_{\alpha}\left(N \cos ^{-1} x\right)\right|
\end{aligned}
$$

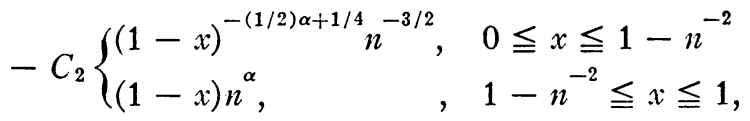

where $N=n+\frac{1}{2}(\alpha+\beta+1)$. Using (1.71.7) and (1.71.1), pp. 14-15 of [5] to estimate the Bessel function in (2.3) shows that there are positive constants, $C_{1}$ and $C_{2}$, independent of $x$ and $n$, such that for $n \geqq 1$ and $0 \leqq x \leqq 1-n^{-2}$

$$
\begin{aligned}
\left|P_{n}^{(\alpha, \beta)}(x)\right| & \geqq(1-x)^{-(1 / 2) \alpha-1 / 4} n^{-1 / 2} \\
& \cdot\left[C_{1}\left|\cos \left(N \cos ^{-1} x-\frac{\alpha \pi}{2}-\frac{\pi}{4}\right)\right|-\frac{C_{2}}{n(1-x)^{1 / 2}}\right],
\end{aligned}
$$

and for $n \geqq 1$ and $1-n^{-2} \leqq x \leqq 1$

$$
\mid \stackrel{(\alpha, \beta)}{P}_{n}^{(x)}\left(\geqq C_{1} n^{\alpha}-C_{2} n^{\alpha+1}(1-x)^{1 / 2} .\right.
$$

Now define

$$
k_{n}(x, y)=\sum_{i=0}^{n} \frac{P_{i}^{(\alpha, \beta)}(x) P_{i}^{(\alpha, \beta)}(y)}{\int_{-1}^{1}\left[P_{i}^{(\alpha, \beta)}(x)\right]^{2}(1-x)^{\alpha}(1+x)^{\beta} d x}
$$

and let

$$
\begin{gathered}
h_{1}(n, x, y)=(n+1) P_{n}^{(\alpha, \beta)}(x) P_{n}^{(\alpha, \beta)}(y), \\
h_{2}(n, x, y)=\frac{n\left(1-y^{2}\right) P_{n}^{(\alpha, \beta)}(x) P_{n-1}^{(\alpha+1, \beta+1)}(y)}{x-y}
\end{gathered}
$$

and 


$$
h_{3}(n, x, y)=h_{2}(n, y, x) .
$$

Following the procedure used by Pollard on pp. 357-8 of [3] and using (4.3.3), (4.5.2), (4.5.5) and (4.21.7) on pp. 68, 71, 72 and 63 respectively of [5] shows that for $\alpha>-1, \beta>-1$ and $n \geqq 2$ that

$$
k_{n}(x, y)=a_{n} h_{1}+b_{n}\left(h_{2}+h_{3}\right)
$$

where the numbers $a_{n}$ and $b_{n}$ depend only on $n, \alpha$ and $\beta$. Furthermore, the values of the coefficients in the expressions from [5] show that the numbers $\left|a_{n}\right|$ and $\left|b_{n}\right|$ are bounded above by a constant depending only on $\alpha$ and $\beta$.

The following lemma will be needed; it is Lemma 8 of [2]. It is also a simple consequence of the theorem mentioned at the end of $\$ 6$, p. 372 of [1].

Lemma. If $1<p<\infty, r>-1 / p, s<1-1 / p, R<1-1 / p, S>-1 / p$, $r \geqq R$ and $s \leqq S$, then there exists a constant, $C$, independent of $f$, such that

$$
\begin{aligned}
\int_{-\infty}^{\infty} \mid \int_{-\infty}^{\infty} & \left.\frac{f(y)}{x-y}|x|^{r}(1+|x|)^{s-r} d y\right|^{p} d x \\
& \leqq\left.\left. C \int_{-\infty}^{\infty}|f(y)| y\right|^{R}(1+|y|)^{S-R}\right|^{p} d y .
\end{aligned}
$$

3. Proof of Theorem 1 and the corollary. The proof of Theorem 1 for $n \geqq 2$ consists of showing for $i=1,2$ and 3 that

$$
\int_{-1}^{1}\left|\int_{0}^{1} f(y) h_{i}(n, x, y)(1-y)^{\alpha}(1-x)^{a}(1+x)^{b} d y\right|^{p} d x
$$

is bounded by the right side of the conclusion of Theorem 1 ; this is sufficient by (2.10), the fact that $\left|a_{n}\right|$ and $\left|b_{n}\right|$ are bounded and the symmetry of the theorem statement and (2.1). For $n=0$ and 1 it is sufficient to obtain the bound for (3.1) just for $i=1$.

For $i=1$ it is immediate from (2.2) and Hölder's inequality that (3.1) has the desired bound. For $i=2$ and 3 (3.1) is bounded above by a constant times the sum of

$$
\int_{-1}^{-1 / 2}\left(\int_{0}^{1}\left|f(y) h_{i}(n, x, y)\right|(1-y)^{\alpha}(1+x)^{b} d y\right)^{p} d x
$$

and

$$
\int_{-1 / 2}^{1}\left|\int_{0}^{1} f(y) h_{i}(n, x, y)(1-y)^{\alpha}(1-x)^{a} d y\right|^{p} d x .
$$


In (3.2) substitute in the value of $h_{i}$ and replace $|x-y|$ by $\frac{1}{2}$. (2.2) and Holder's inequality will produce the desired bound for (3.2).

For $i=2$ use the definition of $h_{2}$ and (2.2) to show that (3.3) equals

$$
\int_{-1 / 2}^{1}\left|\int_{0}^{1} \frac{(1-y)^{\alpha+1}(1-x)^{a} \phi(x, n) \psi(y, n) f(y) d y}{\left(1-y+n^{-2}\right)^{(1 / 2) \alpha+3 / 4}\left(1-x+n^{-2}\right)^{(1 / 2) \alpha+1 / 4}(x-y)}\right|^{p} d x
$$

where $\phi$ and $\psi$ are bounded functions depending only on their indicated arguments, $\alpha$ and $\beta$. Now make the changes of variable $X=n^{2}(1-x)$ and $Y=n^{2}(1-y)$ and drop $\phi$ and $\psi$ since they will not affect the estimate. (3.4) then becomes

$$
n^{-2 a p-2} \int_{0}^{3 n^{2} / 2}\left|\int_{0}^{n^{2}} \frac{Y^{\alpha+1} X^{a} f(y) d Y}{(1+Y)^{(1 / 2) \alpha+3 / 4}(1+X)^{(1 / 2) \alpha+1 / 4}(X-Y)}\right|^{p} d X .
$$

The lemma then shows that (3.5) is bounded above by

$$
C n^{-2 a p-2} \int_{0}^{n^{2}}\left|f(y) Y^{a}\right|^{p} d Y .
$$

Changing the variable in (3.6) back to $y$ shows that (3.6) has the desired bound.

The estimation of (3.3) for $i=3$ is exactly the same. This completes the proof of Theorem 1 .

The corollary is proved in the usual way using theorem 3.1 .5$, p. 40 of [5]. That theorem shows that given $f(x)$ for which the right side of the conclusion of Theorem 1 is finite, there exist polynomials, $g(x)$, that make $\int_{-1}^{1}\left[|f(x)-g(x)|(1-x)^{a}(1+x)^{b}\right]^{p} d x$ arbitrarily small. By Theorem 1 the same integral with $f(x)-g(x)$ replaced by the difference of their $n$th partial sums is small, and if $n$ is large enough, $g(x)$ equals its $n$th partial sum. Minkowski's inequality then completes the proof.

4. Proof of Theorem 2. Let $f(x)$ be a function for which the right side of the conclusion of Theorem 1 is finite. Let $c_{n}$ be the $n$th coefficient in f's Jacobi series. If Theorem 1 is true, then

$$
\int_{-1}^{1}\left|c_{n} P_{n}^{(\alpha, \beta)}(x)(1-x)^{a}(1+x)^{b}\right|^{p} d x
$$

is bounded by the right side of the conclusion of Theorem 1. Using (4.3.3), p. 68 of [5] and reducing the intervals of integration shows that

$$
\int_{0}^{1}\left|P_{n}^{(\alpha, \beta)}(x)(1-x)^{a}\right|^{p} d x\left|\int_{0}^{1} n f(y) P_{n}^{(\alpha, \beta)}(y)(1-y)^{\alpha} d y\right|^{p}
$$


has the same bound. Using the converse of Holder's inequality then shows that there is a constant, $K$, such that

$$
\begin{aligned}
\int_{0}^{1}\left|n^{1 / 2} P_{n}^{(\alpha, \beta)}(x)(1-x)^{a}\right|^{p} d x \\
\cdot\left[\int_{0}^{1}\left|n^{1 / 2} P_{n}^{(\alpha, \beta)}(y)(1-y)^{\alpha-a}\right|^{q} d y\right]^{p / q} \leqq K .
\end{aligned}
$$

Now (2.4) and (2.5) can be used to obtain lower bounds of the integrais in (4.3). To facilitate this, observe that there is a number, $B$, such that if $1-x$ changes by a factor of $B$ on $[s, t]$ and $[s, t]$ $C\left[0,1-n^{-2}\right]$, then $n \cos ^{-1} s-n \cos ^{-1} t \geqq 2 \pi$. Because of this, the cosine in (2.4) can be replaced by a constant when estimating the integrals in (4.3). Estimating those integrals over $\left[0, \frac{1}{2}\right]$ by use of (2.4) shows that both are bounded below by a positive constant. Therefore, both integrals in (4.2) are bounded above by a constant. If a equals the lower bound given for it in the hypothesis of Theorem $1,(2.4)$ or $(2.5)$ can be used to show that the first integral in (4.3) is not bounded as a function of $n$. The form of the integral shows that if it is not bounded for a particular $a$, it is not bounded for a smaller value of $a$. Similarly, if $a$ is greater than or equal to the given upper bound, the second integral in (4.3) is unbounded. The same reasoning shows that $b$ must satisfy the hypothesis of Theorem

1. This completes the proof of Theorem 2 as it applies to Theorem 1.

The fact that Theorem 2 is true for the corollary follows immediately from the preceeding and the Banach-Steinhaus theorem.

\section{REFERENCES}

1. G. H. Hardy and J. E. Littlewood, Some more theorems concerning Fourier series and Fourier power series, Duke Math. J. 2 (1936), 354-382.

2. B. Muckenhoupt, Mean convergence of Hermite and Laguerre series. II, Trans. Amer. Math. Soc. (to appear).

3. H. Pollard, The mean convergence of orthogonal series. II, Trans. Amer. Math. Soc. 63 (1948), 355-367.

4. - The mean convergence of orthogonal series. III, Duke Math. J. 16 (1949), 189-91.

5. G. Szegö, Orthogonal polynomials, Rev. ed, Amer. Math. Soc. Colloq. Publ., vol. 23, Amer. Math. Soc., Providence, R. I., 1959.

6. G. M. Wing, The mean convergence of orthogonal series, Amer. J. Math. 72 (1950), 792-807.

RUtgers UNiversity AND

The Institute for Advanced Study 